Document downloaded from:

http://hdl.handle.net/10251/104823

This paper must be cited as:

Cardós, M.; Guijarro, E.; Babiloni, E. (2017). On the estimation of on-hand stocks for basestock policies and lost sales systems and its impact on service measures. International Journal of Production Research. 55(16):4680-4694. doi:10.1080/00207543.2017.1279759

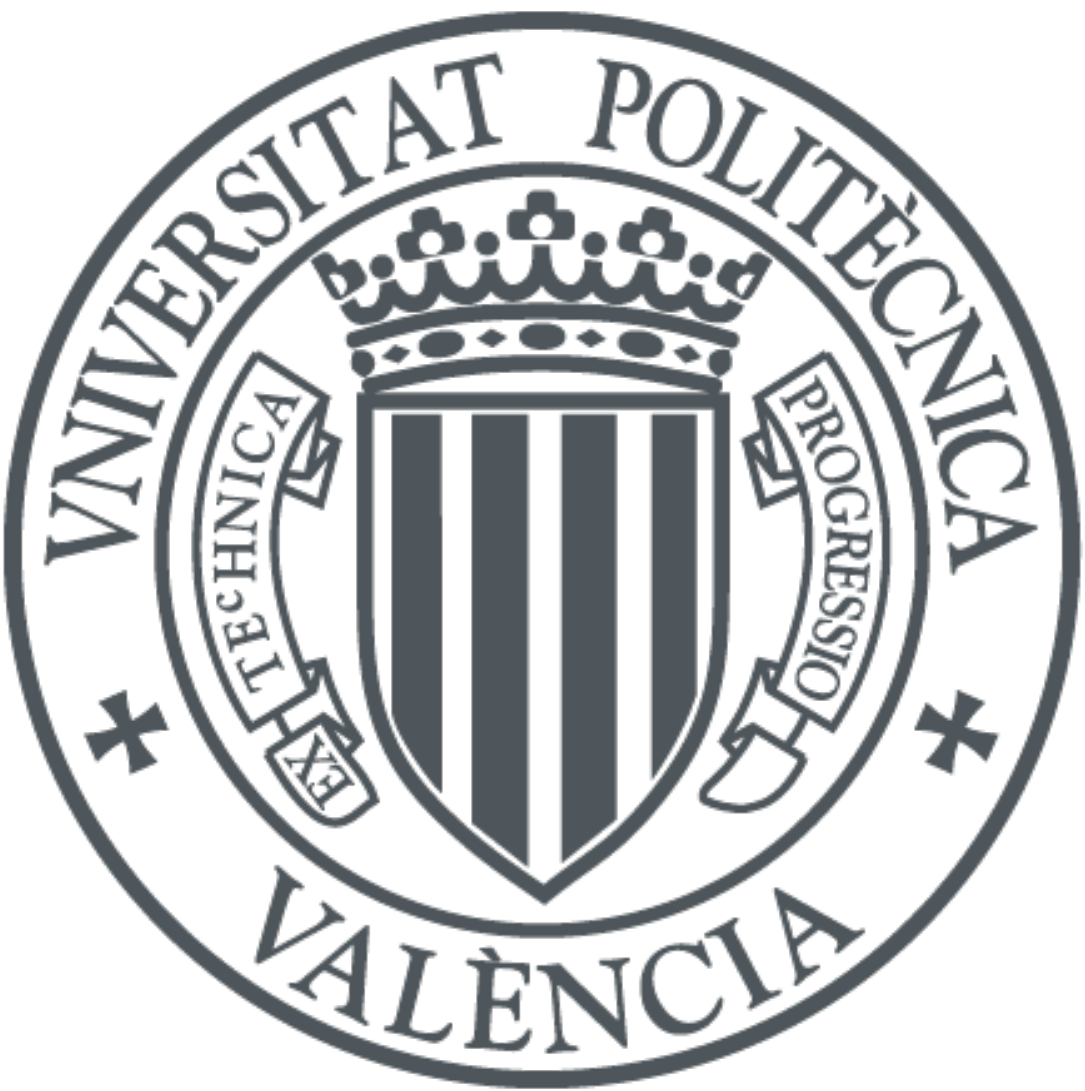

The final publication is available at

https://doi.org/10.1080/00207543.2017.1279759

Copyright Taylor \& Francis

Additional Information 


\title{
On the estimation of on-hand stocks for base-stock policies and lost sales systems and its impact on service measures
}

\begin{abstract}
This paper focuses on computing on-hand stock levels at the beginning of a replenishment cycle for a lost sales inventory system with periodic reviews and discrete demand. A base-stock policy is used for replenishments. The literature provides an Exact method which requires a huge computational effort, and two closed-form approximate methods that arise from the backordering case, the Non-stockout and the Bijvank\&Johansen. In this paper we propose three new and closed-form approaches that explicitly consider the lost sales assumptions: the Adjusted Non-stockout, the Polar Opposite and the 1-Step methods. Existing and proposed methods are evaluated in terms of their accuracy when computing the cycle service level and the fill rate. In this sense, results show that the Bijvank\&Johansen and 1-Step methods provide similar performance but present different behaviours in terms of under or over estimating service measures that have different implications on the design of stock policies.
\end{abstract}

Keywords: inventory; periodic review; lost sales; on-hand stock; discrete demand

\section{Introduction}

When an item is temporarily out of stock, there are two extreme control procedures: (i) backordering unfulfilled demand and fulfilling it as soon as the replenishment order arrives or (ii) losing the entire unfilled demand. Although the problem of lost sales was formulated more than 50 years ago by (Karlin and Scarf 1958), inventory research has traditionally focused on systems where excess demand is backordered. This is mainly because it is much harder to formulate the characteristics of an optimal policy when demand is lost rather than backordered (Bijvank et al. 2014; Zipkin 2008a; Zipkin 2008b). However, the assumption of excess demand being lost is of practical importance in sectors where customers are impatient and they will go to other sources to satisfy their requirements if they do not find the product. Examples are highly competitive sectors such as retail (Gruen, Corsten, and Bharadwaj 2002), 
machinery spare parts, service sector (Diels and Wiebach 2011) or on-line commerce (Breugelmans, Campo, and Gijsbrechts 2006). Hence, having implementable and appropriate expressions to design inventory policies for the lost sales case becomes necessary for practitioners.

One of the challenges in lost sales models is the computation of on-hand stock levels that are necessary to characterize base-stock inventory systems. For the inventory managers, this knowledge is necessary to determine service levels and to establish the control parameters of the inventory policy that will lead to determine total costs of the system. Backordering models use the inventory position (i.e. net stock plus stock on order) as an indicator of the inventory status. The net stock level is the on-hand inventory level minus any backorders. This means that negative values represent stockouts. In contrast, lost sales models do not allow negative net stocks such that the inventory position is computed by means of the on-hand stock. Note that, in this case, the inventory position does not decrease when a stockout takes place (Bijvank and Vis 2011). Therefore it is necessary to know the probability distribution for the on-hand stock level at a crucial point in time, just after an order delivery (we refer to this time point as the beginning of the replenishment cycle). A new replenishment cycle starts just after a replenishment order arrives (a replenishment cycle is defined as the time between two consecutive order deliveries), so it is important to know what is the onhand stock level at the beginning of the cycle to satisfied future demands. This paper focuses on this issue for the lost sales system, discrete demands and periodic review, which is quite straightforward in backordering models but is much more complex when dealing with lost sales models.

In the literature we find only a few papers that deal with the problem of how to compute the on-hand stock levels in the context of lost sales. (Cardós, Miralles, and Ros 
2006) propose an exact method to compute the on-hand stock levels to compute the cycle service level in a discrete demand context. In this approach, which we refer to as Exact Method below, the probability transition matrices of the on-hand stock levels are computed from the beginning of each cycle to its end, and whose convergence gives the on-hand probability vector at the beginning of the cycle. (Cardós and Babiloni 2011) propose an approximation of the cycle service level based on approximating on-hand stock levels at the beginning of the cycle assuming that there are no stockouts during the lead time so that the probability vector of the on-hand stock levels at the beginning of the cycle is computed as in the backordering case. This method, which we call NonStockout henceforth, can be used for any discrete demand distribution. (Bijvank and Johansen 2012) study optimal replenishment policies and derive an approximation procedure to compute the average on-hand stock when demand is (compound) Poisson. The authors develop a closed-form expression to compute the probability of on-hand stocks as in the backordering case by means of a correcting factor in order to avoid negative net stocks. This approach is referred to as Bijvank\&Johansen from here on.

These works are not directly focused on the computation of the on-hand stock probabilities, but they propose several methods to calculate them in order to analyze lost sales inventory systems (either through costs or service level). The Exact Method is very useful for reference purposes; however its computation requires a huge effort and may be time consuming. For this reason, its implementation in practical environments may be rejected by practitioners as, from a practical perspective, 'an understandable decision rule that improves somewhat on current conditions is almost certainly better than the optimal solution that is neither understood nor accepted by management' (Silver 2008). The impact of using the Non-Stockout and the Bijvank\&Johansen 
methods to approximate the probability vector of the on-hand stock levels at the beginning of the cycle will be also evaluated.

The objective of this paper is to derive and evaluate procedures to compute onhand stock levels at order delivery, which are specifically targeted at the lost sales assumption, and any discrete demand distribution that can also be easily implemented in practical environments. To this end we firstly derive three different closed-form expressions to approximate the probability distribution of the on-hand stock at the beginning of the replenishment cycle. Secondly, we state the importance of accurate estimation of the on-hand stock levels by means of evaluating the impact of using approximate methods on the computation of two service measures: the cycle service level and the fill rate. Thirdly we illustrate, through the use of a suitable example, the practical implications of using any of the approximate methods when determining the base-stock level of the policy based on a target fill rate.

The rest of the paper is organized as follows. Section 2 presents the basic notation and assumptions of this paper. Section 3 proposes three new and closed-form approaches to approximate the on-hand stock level probabilities at the beginning of the cycle. Section 4 illustrates the performance of the new approximation procedures proposed in this paper and compares them to existing procedures in terms of the impact on service measures. Section 5 shows an illustrative example of the practical implications of using the approximations derived in this paper when determining the base-stock level. Finally, Section 6 highlights the most relevant conclusions of this research and presents directions for further research.

\section{Notation and Assumptions}

In general, periodic review policies place replenishment orders every $R$ fixed time periods such that the inventory position reaches the base-stock level $S$. The order is 
received $L$ time periods later. Figure 1 shows an example of the evolution of the onhand stock, the net stock and the inventory position in a periodic review system. The notation in Figure 1 and in the rest of the paper is as follows:

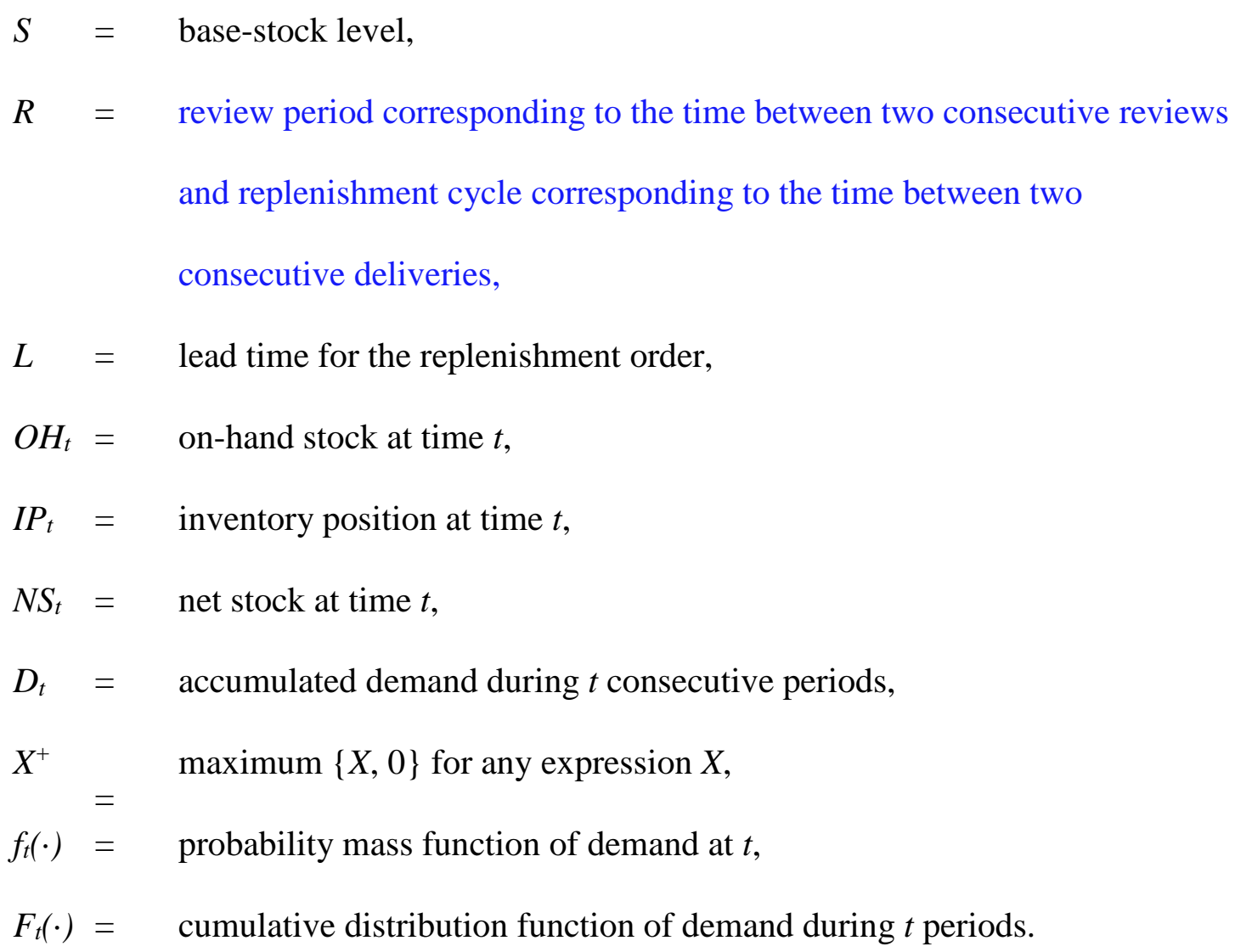

\section{[f]Figure 1 near here[/f]}

General assumptions of this paper are: (i) time is discrete and is organized in a numerable and infinite succession of equally spaced instants; (ii) the lead time, $L$, and the review period, $R$, are constant and known; (iii) there is never more than one order outstanding, this requirement is fulfilled as long as $L<R$; (iv) the replenishment order is added to the on-hand stock $L$ instants after been launched; (v) demand during a period is fulfilled with the on-hand stock at the beginning of the period; (vi) the demand process is considered stationary and i.i.d., and defined by any discrete distribution function; and (vii) unfilled demand is lost. Note that assumption (iii) is widely used both in the literature and in practice since if it is not the case the numerical difficulties are 
insurmountable (Schneider 1981), which is explained in detail by (Hadley and Whitin 1963). In practice, this assumption applies in common situations such as replenishments of a store from a general warehouse in the retail sector where backlog is not allowed. On the other hand, the assumption of stationary and i.i.d. demand is also a common assumption in inventory research that can be extended for the non-stationary demand scenario when forecast errors of the demand are i.i.d.

\section{Proposed Methods}

\subsection{Adjusted Non-stockout approximation}

This first approach proposed is based on the Non-stockout approximation which assumes that there are no stockouts during $L$. We follow the same rationale but modify it to force the sum of probabilities equal to 1 . The on-hand stock balance at order delivery, can be easily computed as

$$
O H_{R}=\left[O H_{R-L}-D_{L}\right]^{+}+S-O H_{R-L} \gg O H_{R-L}-D_{L}+S-O H_{R-L}=S-D_{L}
$$

and then

$$
P\left(O H_{R}=j\right)=P\left(j=S-D_{L}\right)=P\left(D_{L}=S-j\right)=f_{L}(S-j) \quad \forall j \in[0, S]
$$

Note that using expression (2) can lead to the situation in which the sum of the components of the probability vector of the on-hand stock levels at order delivery is not 1 when $D_{L}>O H_{R-L}$. To overcome this shortcoming, we propose adjusting the probability of not having any on-hand stock at the beginning of the cycle, i.e. $P\left(\mathrm{OH}_{\mathrm{R}}=0\right)$. This situation entails that: (i) the on-hand stock at the review moment is exactly equal to the base-stock level, i.e. $O H_{R-L}=S$ and (ii) the demand during the replenishment cycle is equal or greater than the on-hand stock at the review moment, 
i.e. $D_{L} \geq S$ (see Figure 1.b). However, computing $P\left(O H_{R}=0\right)$ through expression (2) is the same as assuming $D_{L}=S$. We adjust $P\left(O H_{R}=0\right)$ computing it as the complement of the sum of probabilities of $O H_{R}>0$, so that cases where $D_{L}>S$ are included. Then, the adjusted vector is defined as

$$
\overline{P\left(O H_{R}\right)} \text { » é } F_{L}(S-1) \quad f_{L}(S-1) \quad \cdots \quad f_{L}(0) \grave{\iota}
$$

the sum of whose probabilities is 1 .

Note that this approach is the same as Bijvank\&Johansen when the correcting factor is set to 1 .

\subsection{Polar Opposites Approximation}

The approximation derived in the previous Section considers the most favourable situation found when managing inventories: there are no stockouts during $L$, i.e. $O H_{R-L} \geq D_{L}$ (which we refer to as NOOS case). On the other hand, the most unfavourable situation is being always out of stock during the replenishment cycle, which implies that $O H_{R-L}<D_{L}$ (referred to as OOS case henceforth). In this Section we propose a closed-form approximation (named Polar Opposites from here on) based on these two opposing cases. First of all, we need to compute the probability on-hand stock vector that results from the NOOS and the OOS cases. After that, we weigh both vectors with their respective probabilities of occurrence, i.e.

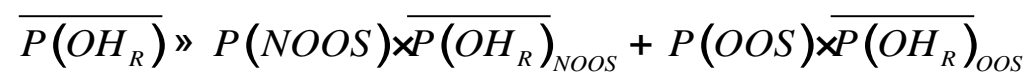

where ${\overline{\mathrm{P}\left(\mathrm{OH}_{\mathrm{R}}\right)_{\mathrm{NOOS}}}}_{=\overline{P\left(\mathrm{OH}_{\mathrm{R}}\right)}}$ from expression (3).

In the OOS case the on-hand stock balance just after the order arrives is

$$
O H_{R}=\left[O H_{R-L}-D_{L}\right]^{\dagger}+S-O H_{R-L}=S-O H_{R-L}
$$


where the on-hand stock balance at the review is

$$
O H_{R-L}=\left[O H_{0}-D_{R-L}\right]^{+}
$$

At this point, we make the following assumptions: (i) $O H_{0}=S$ and (ii) $D_{R-L} \leq O H_{0}$ and therefore the on-hand stock at the review is positive or equal to zero, i.e. $O H_{R-L} \geq 0$. Under these assumptions:

$$
O H_{R} \approx S-\left(S-D_{R-L}\right)=D_{R-L}
$$

and therefore the probability of the on-hand stock at order delivery is

$$
P\left(O H_{R}=j\right) \approx\left\{\begin{array}{lr}
f_{R-L}(j) & 0 \leq j<S \\
1-F_{R-L}(S-1) & j=S
\end{array}\right.
$$

Hence,

$$
\overline{P\left(O H_{R}\right)_{O O S}} \text { » éd } e_{R-L}(0) \quad f_{R-L}(i) \cdots 1-F_{R-L}(S-1) \grave{u}
$$

Then, all that is left is to know the probability of having no stockouts during $L$ :

$$
P(N O O S)=P\left(O_{R-L}^{3} D_{L}\right) \gg P\left(S-D_{R-L}^{3} D_{L}\right)=P\left(D_{R} £ S\right)=F_{R}(S)(10)
$$

and complementary

$$
P(O O S)=1-F_{R}(S)
$$

\subsection{1-Step Approximation}

This method is based on modelling the on-hand stock evolution as an ergodic Markov chain with a set of states $\{0,1, \ldots, S\}$. (Cardós, Miralles, and Ros 2006) define 


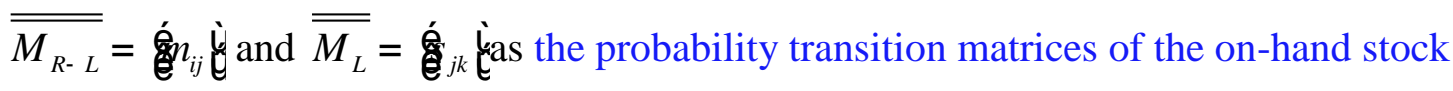
levels from the order delivery to the review and from the review to the next order delivery respectively. Taking into account the on-hand stock balance at $R-L$ (expression (6)), the $\overline{\overline{M_{R-L}}}$ can be computed as

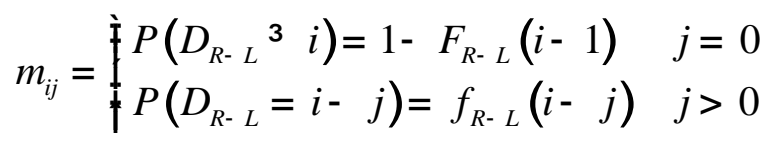

Following the same reasoning, knowing the on-hand stock balance at $R$ is:

$$
O H_{R}=\left[O H_{R-L}-D_{L}\right]^{+}+S-O H_{R-L}
$$

Thus,

$$
s_{j k}=\begin{array}{lr}
\begin{array}{l}
0 \\
P(k=S-j)=P\left(D_{L}^{3} j\right)=1-F_{L}(j-1)
\end{array} & k+j-S<0 \\
P\left(k=j-D_{L}+S-j=S-D_{L}\right)=P\left(D_{L}=S-k\right)=f_{L}(S-k) & k+j-S>0
\end{array}
$$

Then, $\overline{\overline{M_{R}}}=\overline{\overline{M_{R-L}}} \times \overline{\overline{M_{L}}}$ is the transition matrix between two consecutive replenishment cycles. We know that for ergodic Markov chains

$$
\lim _{n \rightarrow \infty} \bar{u} \cdot{\overline{\bar{M}_{R}}}^{n}=\bar{v}
$$

where $\bar{u}$ is an arbitrary vector and $\bar{v}$ is the principal left eigenvector of $\overline{\bar{M}}$, i.e. the probability vector of the on-hand stock levels.

The 1-Step Approximation consists of: (1) initializing the method with an onhand stock level at order delivery equal to $S$ and therefore $\bar{u}=(0,0, \ldots, 1)$; and (2) taking the first steady state probabilities as an estimation of $\bar{v}$. Then, 


$$
\overline{P\left(O H_{R}\right)} \approx \bar{u} \cdot \overline{\overline{M_{R-L}}} \cdot \overline{\overline{M_{L}}}
$$

And therefore

$$
P\left(O H_{R}=i\right) \approx\left\{\begin{array}{lr}
f_{R-L}(0) \cdot\left[1-F_{L}(S-1)\right] & i=0 \\
f_{L}(S-i) \cdot F_{R-L}(i-1)+f_{R-L}(i) \cdot\left[1-F_{L}(S+i-1)\right] & 0<i<S \\
f_{L}(0) \cdot F_{R-L}(S-1)+\left[1-F_{R-L}(S-1)\right] & i=S
\end{array}\right.
$$

\section{Numerical illustration and experimental results}

The goal of this Section is twofold. Firstly we present an illustrative example of

the $\overline{P\left(O H_{R}\right)}$ computed with the different approximations proposed in this paper and the methods available in the literature (the Exact Method, the Non-Stockout approach and the Bijvank\& Johansen expression). After that, we present a wide ranging experiment with different demand patterns in order to compare the performance of these approximate methods when they are used to compute two of the service metrics most used: the cycle service level and the fill rate.

\subsection{Computing the probability of the on-hand stock levels with approximate and exact methods: an illustrative example}

We assume that demand is pure Poisson with $\lambda=1, R=5, L=3$ and $S=5$. Results are summarized in Table 1.

\section{[t]Table 1 near here[/t]}

First of all we observe that each method provides a different vector. The pattern also differs from the Exact to the approximate methods. In this example the Exact methods presents the largest probability associated to $i=5$ whereas for the approximate methods the largest probability is for $i=2$ or $i=3$. 
Furthermore the sum of the components of the vector obtained by the NonStockout approach does not equal 1 . This can be explained by the fact that this method adapts the estimation of the on-hand stock level at the beginning of the cycle from the backordering to the lost sales case. As Section 3.1 points out the Non-Stockout assumes $P\left(O_{R}=0\right)=P\left(D_{L}=S\right)$, whereas the NOOS uses $P\left(O H_{R}=0\right)=P\left(D_{L} \geq S\right)$. So the difference between 1 and 0.916 is the probability of $D_{L}>S$. In the case of Bijvank\&Johansen negative net stocks are avoided by a correcting factor that leads to a negative probability when $i=0$.

After this analysis the question is how to measure the performance of approximate methods since comparing the positions of the vector does not seem to be very useful in practice. As has been explained in the Introduction, the on-hand probability vector at the beginning of the cycle is needed to estimate customer service metrics such as the cycle service level $(\alpha)$ and the fill rate $(\beta)$. Then, a way to measure the performance of the approximate methods is by analysing the impact of using them when estimating $\alpha$ and $\beta$. The next Section is dedicated to this issue.

\subsection{Impact of how to compute the probability of the on-hand stock levels on the estimation of service measures}

\subsubsection{Experiment design}

With the aim of covering as many realistic situations as possible, we consider as input data different demand patterns that cover the smooth, intermittent, erratic and lumpy categories suggested by (Syntetos, Boylan, and Croston 2005), as shown in Figure 2. For the purpose of this experiment, we select the Poisson and the Negative Binomial distributions. The latest can fulfil the four demand categories mentioned above and can also be understood as a compound Poisson distribution. Furthermore, an 
extensive range of values for $R, L$ and $S$ is selected with the aim of providing realistic values of $\alpha$ and $\beta$ (at least from 0.5 to 0.99 ). Table 2 presents the set of data, whose feasible combination results in 12,348 cases (excluding cases where $L \geq R$ ). Then, the onhand probability vector $\overline{P\left(O H_{R}\right)}$ is calculated by applying, on one hand, the current methods available in the literature: the Exact, the Non-stockout and the Bijvank\&Johansen methods and, on the other hand, the new approaches developed in this paper: the Adjusted Non-stockout (expression (3)), the Polar Opposites (expression (4)) and the 1-Step methods (expression (17)). Once the $\overline{P\left(O H_{R}\right)}$ per method is obtained, we compute $\alpha$ and $\beta$ by means of the following expressions:

$$
\begin{gathered}
\alpha=\sum_{i=0}^{S} P\left(O H_{R}=i\right) \cdot \frac{F_{R}(i)-F_{R}(0)}{1-F_{R}(0)} \\
\beta=1-\frac{\sum_{i=0}^{S} P\left(O H_{R}=i\right) \cdot \sum_{j=i+1}^{\infty}(j-i) \cdot f_{R}(j)}{\sum_{j=1}^{\infty} j \cdot f_{R}(j)}
\end{gathered}
$$

\section{[f]Figure 2[/f]}

Note that both expressions are exact and derived for the lost sales case and discrete demand patterns (Cardós, Miralles, and Ros 2006; Guijarro, Cardós, and Babiloni 2012). Therefore, deviations depend only up on the method used to estimate the on-hand stock probability vector. We obtain the absolute errors for $\alpha$ and $\beta$ as the difference between the Exact Method and the corresponding approximate approach. For this analysis we only retain the cases for which service measures are between 0.50 and 0.99 (Table 3 and Table 4). Furthermore it was carried out using the aggregate data, since in a previous analysis we realized that the performance of all the methods is similar for each demand category and random variable. 


\section{[t]Table 2 near here[/t]}

\subsubsection{Results and discussion for $\alpha$}

Figure 3 compares the Exact and the approximate methods. It shows that: (i) all approximations show good performance for high values of $\alpha$. (ii) The Non-stockout and the Adjusted Non-stockout approximation exhibit the same behaviour because expression (18) does not take into account the $P\left(O H_{R}=0\right)$. (ii) The Non-stockout, Adjusted Non-stockout, Polar Opposite and 1-Step approximations show only positive deviations and therefore underestimate $\alpha$. This fact can be explained if we come back to the rationale behind these approximations. Non-stockout and the Adjusted Non-stockout assume that there are no stockouts during $L$, thus these approximations underestimate the on-hand stock level at the beginning of the cycle and therefore the cycle service level. The Polar Opposites method neglects the possibility of being out of stock before reaching the review instant, which entails an underestimation of the on-hand stock level and therefore of the service measures. The 1-Step approach arises from the assumption that the on-hand stock level in the first state is equal to $S$, which is the maximum level possible. Therefore the next state tends to underestimate the on-hand stock level and the service level is underestimated. (iii) The Bijvank\&Johansen method shows positive and negative deviations, i.e. it underestimates but also overestimates $\alpha$. Based on the same distribution pattern of the on-hand stock level as in the backordering case, this method modifies these probabilities to avoid negative net stocks and by applying Little's Law in order to estimate properly the average on-hand stock. Deviations of this method come from situations in which the proposed inventory profile is significantly different from the real inventory profile. Unfortunately these circumstances are not easy to recognize for an inventory manager. This leads to underestimation but also overestimation of the 
on-hand stock levels and therefore the service metric may be underestimated but also overestimated. (iv) As expected, the behaviour of the Polar Opposites approximation is better in extreme situations (i.e. $\alpha$ near to 0 or 1 ), however its deviations are greater when $0.3 \leq \alpha \leq 0.7$.

\section{[f]Figure 3 near here[/f]}

Table 3 presents the maximum, minimum, average and standard deviations of errors. As well as the results that we have already discussed above, we can see that: (i) all the approximations show very small deviations when $\alpha$ is near 1; (ii) the Polar Opposites shows the largest deviations compared to the simplest Non-stockout, and therefore is not a suitable approximation in any case; (iii) Bijvank\&Johansen exhibits the lowest average and standard deviation of errors and for this reason is the best method from this point of view, with 1-Step second ranked; (iv) Bijvank\&Johansen method may overestimate but also underestimate $\alpha$, so, if used to determine the basestock level, unexpected stockout occasions may occur; and (v) the 1-Step approach also presents very low average and standard deviations and underestimates $\alpha$. Therefore the 1-Step method can be considered the most suitable approach to design inventory policies without the risk of underestimating the base-stock level.

\section{[t]Table 3 near here[/t]}

\subsubsection{Results and discussion for $\beta$}

Figure 4 illustrates the performance of the Exact fill rate versus approximate expressions. It can be observed that: (i) similar to the case of $\alpha$, all approximate methods properly estimate the fill rate when it is close to 1; (ii) the Adjusted Nonstockout, the Polar Opposites and the 1-Step approximation always underestimate $\beta$ whereas the Non-stockout method underestimates but also overestimates $\beta$. The 
explanation made in the previous section regarding the positive deviations shown by the Adjusted Non-stockout, the Polar Opposites and the 1-Step approximation also apply to the fill rate estimation. However, the results of the Non-Stockout are different to those obtained for $\alpha$. This can be explained given that when calculating the fill rate with expression (19), the probability that the stock is equal to zero is taken into account, showing that the negative deviations that appear in the calculation are due to this. Finally, (iii) Bijvank\&Johansen method always overestimates $\beta$.

\section{[f]Figure 4 near here[/f]}

Table 4 shows the maximum, minimum, average and standard deviations of fill rate errors. In addition to the results that we have commented upon above, we can observe that: (i) the 1-Step shows robust performance: only shows positive deviations which are very small (less that $1.24 \%$ on average) when $\beta>0.85$; (ii) the performance of the Polar Opposites and the Adjusted Non-stockout is quite similar, with the Adjusted Non-stockout being mathematically simpler and more accurate for $\beta>0.65$. (iii) Bijvank\&Johansen and 1-Step methods show very similar average deviations. Therefore, we reach to the same conclusion as in the case of $\alpha$.

\section{[t]Table 4 near here[/t]}

\section{Practical implications of the probability computation method of on-hand stock levels on the design of inventory policies}

This Section illustrates practical implications of determining the base-stock level, $S$, using the approximate on-hand approaches and the method based on meanvalue analysis (referred to as MVA further on) proposed by (Bijvank and Vis 2012) which is the best approach known in the literature to set the base-stock level when there is a service level constraint in a lost sales inventory system. Table 5 presents an example of the different values of $S$ for different fill rates when demand is intermittent 
and modeled by a pure Poisson with $\lambda=1, R=20$ and $L=10$. It shows that the $S$ that we obtain when using the Non-stockout, Adjusted Non-stockout, Polar Opposites and 1Step approaches is greater than the exact $S$, except for the case of $\beta=0.99$ in which case the Non-stockout is one unit above the exact $S$. This type of performance in the determination of base-stock levels can lead to increased on-hand inventory levels but assures the system reaches the target fill rate. However, this is not the case when using the Bijvank\&Johansen and the MVA methods that underestimate the base-stock level for any fill rate. Therefore, this performance implies that the target fill rate could not be achieved and as a result the system is less protected against stockout occasions than it might be expected. For example when a target fill rate is equal to 0.8 , the exact method provides a base-stock level equal to 24 units whereas when using the Non-stockout, the Adjusted Non-stockout, the Polar Opposites and the 1-Step, the base-stock level is 27, 27, 28 and 27 respectively. This type of deviations implies an increase of the holding costs but guarantees the target service level. However, the Bijvank\&Johansen method sets a base-stock level equal to 18 units and the MVA approach equal to 22 which implies a fill rate lower than the target. Comparing the performance of all the methods, it can be observed that the base-stock level obtained by the MVA method is in almost all the cases the closest to the exact one, except when the target fill rate is greater than 0.9 in which cases the 1-Step is the closest one. Furthermore, Adjusted Non-stockout and Polar Opposites methods show the same results as the 1 -Step method when $\beta \geq 0.85$.

\section{[t]Table 5 near here[/t]}

\section{Summary and Conclusions}

This paper focuses on the estimation of the on-hand stock level at the beginning of the cycle for the lost sales system, discrete demand and periodic review. As is well 
known, characterizing optimal policies for the lost sales case is quite complex. However, there are many real life situations where backordering models cannot be applied and it becomes necessary to have appropriate methods developed specifically for the lost sales assumption. In inventory systems the demand is satisfied with the available stock at the beginning of the cycle, i.e. just after the order arrives. The problem of computing the on-hand stock level at the beginning of the cycle in lost sales systems arises from the fact that it cannot be computed as the base-stock level minus the demand during the lead time (as in backordering models), thus finding the on-hand stock probability vector becomes a challenge. Therefore, the key question in lost sales models is to accurately know the on-hand stock steady probability vector which is required to compute service measures and design optimal inventory policies.

In the literature we found three methods to estimate the probability vector associated to every feasible value of the on-hand stock: one Exact Method, which is not a closed-form expression and requires a huge computational effort for its implementation in practical environments, and two closed-form approximate expressions (Non-Stockout and Bijvank\&Johansen methods) that adapt backorder formulas to the lost sales case. This paper suggests three additional approximate closedform estimations that are specifically based on the characteristics of the lost sales system.

In order to analyze the performance of the existing methods and the new approaches proposed in this paper (Adjusted Non-stockout, Polar Opposites and 1-Step), we firstly compare the on-hand probability vector that results from each method through an illustrative example (Table 1). We observe that when using Non-Stockout the components of the vector do not add up to 1 . Furthermore, the Bijvank\&Johansen method provides negative probabilities for zero on-hand stock for low service levels. 
The approaches proposed in this paper do not suffer from any of these drawbacks. Secondly, we design a large experiment with the aim of analyzing the impact of using the approximate methods to compute the cycle service level $(\alpha)$ and the fill rate $(\beta)$. With respect to $\alpha$, the Bijvank\&Johansen method shows the best results in terms of the average and standard deviations, followed by the 1-Step. However, the former overestimates and also underestimates the cycle service level whilst the latter only underestimates. Therefore the 1-Step should be preferred when the risk of not reaching the target cycle service level cannot be accepted. With respect to the fill rate, the 1-Step and the Bijvank\&Johansen methods are the best performers with really small deviations. However, 1-Step always underestimates $\beta$ whereas Bijvank\&Johansen always overestimates this service measure.

Regarding the other approaches, the Polar Opposites does not offer any advantage over the Adjusted Non-stockout which is simpler and more accurate for both service measures. Both approaches only underestimate $\alpha$ and $\beta$. The Non-stockout and the Adjusted Non-stockout show the same performance for $\alpha$ (given that this metric does not consider the probability of zero on-hand stock), but with respect to $\beta$ the former shows positive and negative values. The drawbacks of the Non-stockout are overcome by the Adjusted Non-stockout approach.

Section 5 presents an illustrative example to show the risks associated with using an approximate on-hand approach to determine the base-stock level based on a target fill rate including the MVA approach. In this sense, the Adjusted Non-stockout, Polar Opposites and 1-Step methods can lead to an increase of the stock level, albeit with a guarantee of achieving the target service level. The Bijvank\&Johansen and the MVA method presents only negative deviations that leads to set base-stock levels lower than that necessary to achieve the target fill rate. Despite MVA method is in almost all 
the cases the closest to the exact one (except when the target fill rate is greater than 0.9) the inventory system would be less protected against stockouts than they might expect, as shown in Table 5.

A logical further extension of this work consists of designing a huge experiment in order to deeply analyze the approaches presented in this paper and the approaches available in the literature for determining base-stock levels in the context of lost sales and discrete demands. Another important avenue for future research in this field will consist of relaxing the assumptions of stationary and i.i.d. demand and analyzing the performance of the proposed approximations in that case.

\section{Acknowledgements}

We gratefully acknowledge the help of Dr. John Boylan, University of Lancaster, and Dr. Marco Bijvank, University of Calgary, for providing useful comments and feedback to help improve this work. We also express our gratitude to the anonymous referees of this paper whose comments really have contributed to improve the quality of our work. All errors remain ours.

This work was supported by the European Regional Development Fund and Spanish Government (MINECO/FEDER, UE) under the project with reference DPI2015-64133-R.

\section{References}

Bijvank, M., W. T. Huh, G. Janakiraman, and W. Kang. 2014. "Robustness of Orderup-to Policies in Lost-Sales Inventory Systems”. Operations Research 62 (5): 1040-1047

Bijvank, M., and S. G. Johansen. 2012. "Periodic review lost-sales inventory models with compound Poisson demand and constant lead times of any length”. 
European Journal of Operational Research 220 (1): 106-114.

doi:10.1016/j.ejor.2012.01.041

Bijvank, M., and I. F. A. Vis. 2011. "Lost-sales inventory theory: A review”. European Journal of Operational Research 215 (1): 1-13. doi:10.1016/j.ejor.2011.02.004

Bijvank, M., and I. F. A. Vis. 2012. “Lost-sales inventory systems with a service level criterion”. European Journal of Operational Research 220(3): 610-618

Breugelmans, E., K. Campo, and E. Gijsbrechts. 2006. “Opportunities for active stockout management in online stores: The impact of the stock-out policy on online stock-out reactions”. Journal of Retailing 82 (3): 215-228. doi:10.1016/j.jretai.2006.05.001

Cardós, M., and E. Babiloni. 2011. "Exact and approximate calculation of the cycle service level in periodic review inventory policies”. International Journal of Production Economics 131 (1): 63-68. doi:10.1016/j.ijpe.2010.05.012

Cardós, M., C. Miralles, and L. Ros. 2006. “An exact calculation of the cycle service level in a generalized periodic review system”. Journal of the Operational Research Society 57 (10): 1252-1255.

Diels, J. L. and N. Wiebach. 2011. Customer reactions in Out-of-Stock situations: Do promotion-induced phantom positions alleviate the similarity substitution hypothsis? Berlin: SFB 649 Discussion paper 2011-021

Gruen, T. W., D. Corsten, and S. Bharadwaj. 2002. Retail Out-of-Stocks: A Worldwide Examination of Extent Causes, Rates and Consumer Responses. Washington, D.C.: Grocery Manufacturers of America

Guijarro, E., M. Cardós, and E. Babiloni. 2012. “On the exact calculation of the fill rate in a periodic review inventory policy under discrete demand patterns”. European Journal of Operational Research 218 (2): 442-447. doi:10.1016/j.ejor.2011.11.025

Hadley, G ., and T. M. Whitin. 1963. Analysis of inventory systems.New Jersey: Prentice Hall, Inc.

Karlin, S., and H. Scarf. 1958. Inventory models of the Arrow-Harris-Marschak type with time lag. In Studies in the mathematical theory of inventory and production, Standford, Ca.: Standford University Press

Schneider, H. 1981. "Effect of service-levels on order-points or order-levels in inventory models”. International Journal of Production Research 19 (6): 615631. 
Silver, E. A. 2008. "Inventory Management: An overview, Canadian publications, practical applications and suggestions for future research”. INFOR: Information Systems and Operational Research 46 (1): 15-28. doi:

http://dx.doi.org/10.3138/infor.46.1.15

Syntetos, A. A., J. E. Boylan, and J. D. Croston. 2005. “On the categorization of demand patterns”. Journal of the Operational Research Society 56 (5): 495-503. doi:10.1057/palgrave.jors.2601841

Zipkin, P. 2008a. “Old and New Methods for Lost-Sales Inventory Systems”. Operations Research 56 (5): 1256-1263, ISI:000261236800016

Zipkin, P. 2008b. “On the structure of lost-sales inventory models”. Operations Research 56 (4): 937-944. 